\title{
Limitations of SARC-F as a Screening Tool for Sarcopenia in Patients on Hemodialysis
}

\author{
Keigo Imamura ${ }^{a}$ Shohei Yamamoto ${ }^{a, b}$ Yuta Suzukia,c, d Ryota Matsuzawa \\ Manae Haradac Shun Yoshikoshi ${ }^{a}$ Atsushi Yoshida ${ }^{f}$ Atsuhiko Matsunaga $^{a}$ \\ aDepartment of Rehabilitation Sciences, Kitasato University Graduate School of Medical Sciences, Sagamihara, \\ Japan; bepartment of Epidemiology and Prevention, Center for Clinical Sciences, National Center for Global \\ Health and Medicine, Tokyo, Japan; 'Department of Rehabilitation, Sagami Circulatory Organ Clinic, Sagamihara, \\ Japan; ${ }^{d}$ Department of Advanced Research Course, National Institute of Public Health, Wako, Japan; 'Department \\ of Physical Therapy, School of Rehabilitation, Hyogo University of Health Sciences, Kobe, Japan; fDepartment of \\ Hemodialysis Center, Sagami Circulatory Organ Clinic, Sagamihara, Japan
}

\section{Keywords}

Hemodialysis · SARC-F · Sarcopenia · Screening · Japanese

\begin{abstract}
Introduction: There are limited screening tools for sarcopenia in patients undergoing hemodialysis. This study aimed to investigate the reliability and validity of the SARC-F (Strength, Assistance in walking, Rise from a chair, Climb stairs, and Falls) questionnaire as a screening tool for sarcopenia (defined by the Asian Working Group for Sarcopenia [AWGS2019]) in patients undergoing hemodialysis. Methods: This cross-sectional study enrolled 179 patients (mean age: $66.5 \pm 12$ years, $58 \%$ men) undergoing maintenance hemodialysis 3 times per week at a hemodialysis center in Japan. The SARC-F score, handgrip strength, usual gait speed, sit-to-stand test time, short physical performance battery (SPPB), and appendicular skeletal muscle mass were evaluated. The reliability and validity of the SARC-F were analyzed using receiver-operating characteristic curve, area under the
\end{abstract}

karger@karger.com www.karger.com/nef

Karger $\stackrel{\text { ' }}{5}$

BOPEN ACCESS
(C) 2021 The Author(s)

Published by S. Karger AG, Basel

This is an Open Access article licensed under the Creative Commons Attribution-NonCommercial-4.0 International License (CC BY-NC) (http://www.karger.com/Services/OpenAccessLicense), applicable to the online version of the article only. Usage and distribution for commercial purposes requires written permission. curve (AUC), and sensitivity/specificity analyses. Results: There were $49(27.4 \%)$ patients with sarcopenia. Patients with SARC-F $\geq 4$ (59 patients, 33.0\%) had poorer grip strength, lower SPPB score, and slower gait speed than those with $S A R C-F<4$, while the skeletal muscle mass index did not differ significantly between the two groups. The sensitivity and specificity values of the SARC-F for identifying sarcopenia were $42.9 \%$ and $70.8 \%$, respectively, while those for identifying severe sarcopenia were $66.7 \%$ and $72.3 \%$, respectively. The AUCs of SARC-F were 0.57 for sarcopenia and 0.70 for severe sarcopenia. Discussion/Conclusion: The SARC-F alone is an inadequate screening tool for sarcopenia in patients undergoing hemodialysis. It should be used in combination with objective assessment measures, rather than as a first-step screening tool, to diagnose sarcopenia.

(C) 2021 The Author(s)

Published by S. Karger AG, Basel

Keigo Imamura and Shohei Yamamoto contributed equally as first authors.
Correspondence to:

Shohei Yamamoto, ap13338@st.kitasato-u.ac.jp 


\section{Introduction}

Sarcopenia is defined as the loss of skeletal muscle mass, muscle strength, and physical ability associated with aging $[1,2]$. Sarcopenia is associated with a risk of fractures, depression, cognitive impairment, frailty, impaired ability to perform activities of daily living, and mortality in community-dwelling older adults [3-8]. Furthermore, it has been noted that sarcopenia might be more severe in vulnerable populations, such as patients with end-stage renal disease [9]. In patients on hemodialysis, the prevalence of sarcopenia has been reported to be between $12.7 \%$ and $40.0 \%$ [10-13], and it has been associated with adverse events such as mortality $[10,13-$ 15]. Therefore, early identification and intervention for sarcopenia could be essential for effective disease management in patients on hemodialysis.

Sarcopenia diagnosis requires detailed investigations into the muscle strength and quantity. However, in clinical practice, this is challenging due to several reasons, such as a lack of experts (e.g., physiotherapists) and measuring equipment in the dialysis units $[16,17]$. Therefore, there is a need for a simple tool that can help nephrologists in screening patients for sarcopenia in the clinical settings.

Recently, the European Working Group on Sarcopenia (EWGSOP) and the Asian Working Group on Sarcopenia (AWGS) have revised the diagnostic algorithm and some of the diagnostic cutoff values for sarcopenia $[2,18]$. These recommendations, published in the EWGSOP2 and AWGS2019 guidelines, suggest case finding to identify early-stage sarcopenia and screen for sarcopenia. The SARC-F (Strength, Assistance in walking, Rise from a chair, Climb stairs, and Falls) is a simple questionnaire that can be used as an initial screening tool for sarcopenia and for quickly assessing the physical function $[19,20]$. The SARC-F also predicts hospitalization and mortality in community-dwelling older adults and those with cardiovascular disease $[4,21,22]$. Furthermore, the SARC-F has been reported to be highly accurate in identifying the risk of physical limitations in patients on hemodialysis [23]. Therefore, we similarly hypothesized that the SARCF may be useful as a screening tool for sarcopenia.

However, it is unclear whether the SARC-F is useful in screening for sarcopenia (defined by the muscle strength, physical performance, and muscle mass) in patients on dialysis. Therefore, the aim of this study was to validate the availability of the SARC-F as a screening tool for sarcopenia and to investigate the sensitivity and specificity of the SARC-F for sarcopenia (as defined by the AWGS2019).

Limitations of SARC-F Usage in Patients on Hemodialysis

\section{Methods}

\section{Study Design and Population}

This cross-sectional study enrolled clinically stable outpatients who underwent hemodialysis between April 2018 and February 2019 at a single dialysis unit in Japan. A total of 313 outpatients were assessed for eligibility. We excluded patients from further analyses for the following reasons: hospitalization within 3 months before the study $(n=25)$; severe dementia $(n=6)$; refusal to participate in this study $(n=35)$; missing data on the SARC-F $(n=$ $24)$; and missing data on the muscle mass, muscle strength, or physical performance $(n=44)$. Finally, 179 patients on hemodialysis were included (online supplementary Fig. S1, available at www.karger.com/doi/10.1159/000518810). All participants were on dialysis for at least 3 months and were able to provide written consent. This study was performed in accordance with the ethical principles of the Declaration of Helsinki and was approved by the institutional research ethics committee.

\section{Patient Characteristics}

Data on the demographic factors (age, sex, body mass index [BMI], and hemodialysis vintage), primary kidney disease (glomerulonephritis/cystic kidney disease, diabetes, hypertension, others, and unknown), comorbidities, and biochemical results were collected from the medical records updated during the weeks in which the patients participated in the study. A comorbidity index [24], which was developed for patients on dialysis, was used to quantify the comorbidities. It included the primary causes of end-stage renal disease, atherosclerotic heart disease, congestive heart failure, cerebrovascular accident/transient ischemic attack, peripheral vascular disease, dysrhythmia, other cardiac diseases, chronic obstructive pulmonary disease, gastrointestinal bleeding, liver disease, cancer, and diabetes. The geriatric nutritional risk index [25] was also calculated as an index of the nutritional condition based on the serum albumin level and BMI. A short, 10-item version of the Center for Epidemiological Studies Depression Screening Index was used to assess the symptoms of depression, which were defined by a score of $\geq 10$ [26]. The Mini-Mental State Examination (MMSE) was used to evaluate the cognitive function. Cognitive impairment was defined by a score less than the cutoff point of 24 [27]. The education levels of the patients were classified into "lower than high school" and "high school or higher."

\section{SARC-F Questionnaire}

The patients were asked to complete the SARC-F questionnaire at the time of the physical function evaluation. The SARC-F is a self-administered questionnaire used to determine the level of difficulty experienced in 5 components comprising the strength, assistance in walking, rising from a chair, climbing stairs, and history of falls; a score of $0-2$ points is assigned for each item (Table 1 ) [19]. The total score ranges from 0 to 10 , with scores of $\geq 4$ points indicating the risk of sarcopenia.

\section{Measurement of Muscle Strength}

Muscle strength was assessed by the handgrip strength, which was measured using a digital dynamometer (TKK 5101 Grip-D; Takei, Tokyo, Japan) with the patients in the sitting position. Maximal isometric voluntary contractions of both hands were measured for $3 \mathrm{~s}$ each, with the elbow joint flexed at $90^{\circ}$. Handgrip 
Table 1. SARC-F score

\begin{tabular}{|c|c|c|}
\hline Component & Question & Scoring \\
\hline Strength & How much difficulty do you have in lifting and carrying $10 \mathrm{lb} ?$ & $\begin{array}{l}\text { None }=0 \\
\text { Some }=1 \\
\text { A lot or unable }=2\end{array}$ \\
\hline Assistance in walking & How much difficulty do you have walking across a room? & $\begin{array}{l}\text { None }=0 \\
\text { Some }=1 \\
\text { A lot, use aids, or unable }=2\end{array}$ \\
\hline Rise from a chair & How much difficulty do you have transferring from a chair or bed? & $\begin{array}{l}\text { None }=0 \\
\text { Some }=1 \\
\text { A lot, or unable without help }=2\end{array}$ \\
\hline Climb stairs & How much difficulty do you have climbing a flight of 10 stairs? & $\begin{array}{l}\text { None }=0 \\
\text { Some }=1 \\
\text { A lot or unable }=2\end{array}$ \\
\hline Falls & How many times have you fallen in the past year? & $\begin{array}{l}\text { None }=0 \\
1-3 \text { falls }=1 \\
\geq 4 \text { falls }=2\end{array}$ \\
\hline
\end{tabular}

strength was measured twice on each side, and the highest value (expressed as the absolute value in kilograms) was used in the analyses.

\section{Assessment of the Physical Performance}

Usual gait speed was measured during a $10-\mathrm{m}$ timed walk. Patients were asked to walk the course at their usual pace and were timed at the middle of a 10- to 16-m walkway. To account for acceleration and deceleration, there was a 3-m distance before and after their assigned walking space. Usual gait speed was measured once and expressed in meters per second.

For the sit-to-stand test, a stopwatch was used to measure how long it took the participant to stand up from a sitting position and sit down again with the arms crossed in front of the chest, 5 times, as quickly as possible. The Short Physical Performance Battery (SPPB), which consists of the usual gait speed, repeated standing up from the chair, and standing balance, was measured according to the established methods [28]. The SPPB score ranged from 0 to $12(0=$ worst and $12=$ best $)$, with a scale of $0-4$ points for each component.

Most patients underwent predialysis muscle strength and physical performance tests. However, those who could be assessed only after dialysis were evaluated when they were in a good physical condition.

\section{Assessment of Muscle Mass}

Muscle mass was assessed before a dialysis session by multifrequency whole-body BIA using InBody S10 (InBody Co., Ltd., Seoul, South Korea), which provided the total and segmental muscle mass through the arms and legs in kilograms. Patients were asked to remain in the sitting position for $10 \mathrm{~min}$ before the test to ensure body fluid balance. The appendicular skeletal muscle mass (ASM) was measured, and the skeletal muscle mass index (SMI) was calculated as ASM $(\mathrm{kg}) /$ height $(\mathrm{m})^{2}$.

\section{Definition of Sarcopenia by the AWGS2019 Criteria}

Sarcopenia was defined using the AWGS2019 criteria [2]. The cutoff values were as follows: low muscle mass was defined as SMI $<7.0 \mathrm{~kg} / \mathrm{m}^{2}$ for men and $<5.7 \mathrm{~kg} / \mathrm{m}^{2}$ for women; low muscle strength was defined as handgrip strength $<28 \mathrm{~kg}$ for men and $<18$ $\mathrm{kg}$ for women; and low physical performance was defined as a gait speed $<1.0 \mathrm{~m} / \mathrm{s}$, sit-to-stand time $\geq 12 \mathrm{~s}$, and/or SPPB $\leq 9$ for both sexes. According to the AWGS2019 criteria, sarcopenia was diagnosed if the patient had a low muscle mass and low muscle strength or low physical performance. Severe sarcopenia was defined as the presence of low muscle mass, low muscle strength, and low physical performance.

\section{Statistical Analysis}

Normally distributed continuous variables were expressed as means \pm standard deviations, and nonnormally distributed continuous variables were presented as medians (interquartile ranges). Categorical variables were expressed as numbers and percentages. The patients were classified into two groups according to the SARC-F score: SARC-F $<4$ and SARC-F $\geq 4$ [29]. Baseline patient characteristics and the prevalence of sarcopenia were compared between the two groups using the unpaired Student's $t$ test, MannWhitney $U$ test, or $\chi^{2}$ test, as appropriate. In addition, we evaluated the sensitivity, specificity, and positive and negative predictive values. The areas under the curves (AUCs) for the SARC-F scores, defined by the AWGS2019 diagnostic criteria, were computed, and receiver operating characteristic curve analysis was performed. Furthermore, the sensitivity, specificity, and positive and negative predictive values of each SARC-F score were calculated to investigate the screening accuracy of SARC-F for sarcopenia (as defined by the AWGS2019). The analyses were performed using Stata version 16.1 (Stata Corp LP, College Station, TX, USA). A two-tailed $p$ value of $<0.05$ was considered to indicate statistical significance. 
Table 2. Characteristics of the study population

\begin{tabular}{|c|c|c|c|c|}
\hline & $\begin{array}{l}\text { Overall } \\
179\end{array}$ & $\begin{array}{l}\text { SARC-F }<4 \\
120\end{array}$ & $\begin{array}{l}\text { SARC-F } \geq 4 \\
59\end{array}$ & $p$ value \\
\hline Age, years & $66.5(12.0)$ & $63.4(11.5)$ & $72.9(10.5)$ & $<0.001$ \\
\hline Men & $104(58.1)$ & $76(63.3)$ & $28(47.5)$ & 0.053 \\
\hline $\mathrm{BMI}, \mathrm{kg} / \mathrm{m}^{2}$ & $21.9(4.2)$ & $22.1(4.2)$ & $21.6(4.4)$ & 0.484 \\
\hline Time on hemodialysis, years & $6.0[2.0-14.0]$ & $7.0[2.3-15.0]$ & $5.0[2.0-13.0]$ & 0.380 \\
\hline Primary kidney disease & & & & 0.232 \\
\hline GN/cystic kidney disease & $58(32.4)$ & $43(35.8)$ & $15(25.4)$ & \\
\hline Diabetes & $60(33.5)$ & $37(30.8)$ & $23(39.0)$ & \\
\hline Hypertension & $14(7.8)$ & $8(6.7)$ & $6(10.2)$ & \\
\hline Others & $26(14.5)$ & $15(12.5)$ & $11(18.6)$ & \\
\hline Unknown & $21(11.7)$ & $17(14.2)$ & $4(6.8)$ & \\
\hline \multicolumn{5}{|l|}{ Comorbidity condition } \\
\hline $\mathrm{CHF}$ & $22(12.7)$ & $11(9.2)$ & $11(18.6)$ & 0.090 \\
\hline Diabetes & $77(44.5)$ & $48(40.0)$ & $31(52.5)$ & 0.149 \\
\hline CVA/TIA & $47(27.2)$ & $19(15.8)$ & $30(50.9)$ & $<0.001$ \\
\hline Comorbidity score & $6.0[4.0-9.0]$ & $5.0[3.0-7.8]$ & $7.0[6.0-10.0]$ & $<0.001$ \\
\hline Hemoglobin, g/dL & $10.9(0.9)$ & $11.0(1.0)$ & $10.9(0.9)$ & 0.661 \\
\hline Albumin, g/dL & $3.8(0.3)$ & $3.9(0.3)$ & $3.8(0.3)$ & 0.032 \\
\hline GNRI $\leq 91$ & $38(21.2)$ & $21(17.5)$ & $17(28.8)$ & 0.119 \\
\hline MMSE $<24$ & $55(30.7)$ & $24(20.0)$ & $31(52.5)$ & $<0.001$ \\
\hline Education level* & & & & $<0.001$ \\
\hline Less than high school & $24(14.4)$ & $8(7.2)$ & $16(28.6)$ & \\
\hline High school or higher & $143(85.6)$ & $103(92.8)$ & $40(71.4)$ & \\
\hline Depressive symptoms & $52(29.1)$ & $30(25.0)$ & $22(37.3)$ & 0.115 \\
\hline Handgrip strength, kg & $24.9(8.5)$ & $27.0(7.9)$ & $20.6(8.2)$ & $<0.001$ \\
\hline Usual gait speed, m/s & $1.1(0.3)$ & $1.3(0.3)$ & $0.9(0.3)$ & $<0.001$ \\
\hline Sit to stand, s & $10.0(4.3)$ & $9.0(3.0)$ & $12.7(5.7)$ & $<0.001$ \\
\hline SPPB score & $12.0[10.0-12.0]$ & $12.0[11.0-12.0]$ & $9.0[6.0-11.0]$ & $<0.001$ \\
\hline Skeletal muscle index, $\mathrm{kg} / \mathrm{m}^{2}$ & $6.9(1.3)$ & $7.0(1.2)$ & $6.6(1.3)$ & 0.047 \\
\hline
\end{tabular}

Values are expressed as mean (standard deviation), $n$ (\%) of patients, or median [interquartile range]. BMI, body mass index; CHF, congestive heart failure; CVA/TIA, cerebrovascular accident/transient ischemic attack; GN, glomerulonephritis; GNRI, geriatric nutritional risk index; MMSE, Mini-Mental State Examination; SPPB, short physical performance battery. ${ }^{*}$ Missing values on education level $(n=12)$.

\section{Results}

\section{Patient Characteristics}

Table 2 shows the characteristics of all participants and the groups stratified by the SARC-F score. The mean age of the study population was $66.5 \pm 12$ years. Furthermore, $58 \%$ of the patients were men, and the median hemodialysis vintage was 6.0 years (interquartile range: $2.0-14.0$ years). There were $59(33.0 \%)$ and $120(67 \%)$ patients in the SARC-F $\geq 4$ and SARC-F $<4$ groups, respectively. The number of patients with sarcopenia according to the AWGS2019 criteria was 49 (27.4\%). Compared to the SARC-F $<4$ group, the SARC-F $\geq 4$ group had older patients, higher comorbidity scores, higher prevalence of cognitive impairment, and greater number of patients with a less than high school level of education. Further- more, the SARC-F $\geq 4$ group had a significantly lower handgrip strength, slower usual gait speed, longer sit-tostand position time, lower SPPB score, and poorer physical performance as compared to the $\mathrm{SARC}-\mathrm{F}<4$ group (all $p<0.001)$. In contrast, there was no significant difference in the prevalence of low SMI between the two groups (Table 3).

\section{Validation of the SARC-F}

The SARC-F and AWGS2019 criteria diagram is shown in Figure 1. When assessed according to the AWGS2019 criteria, the SARC-F $\geq 4$ group comprised 5 and 16 patients with sarcopenia and severe sarcopenia, respectively. In contrast, the SARC-F $<4$ group comprised 20 and 8 patients diagnosed with sarcopenia and severe sarcopenia, respectively. These results showed that 
Table 3. Comparison for the number of patients who met each criterion for sarcopenia

\begin{tabular}{lllll}
\hline & $\begin{array}{l}\text { Overall } \\
179\end{array}$ & $\begin{array}{l}\text { SARC-F }<4 \\
120\end{array}$ & $\begin{array}{l}\text { SARC-F } \geq 4 \\
59\end{array}$ & $p$ value \\
\hline Handgrip strength men $<28 \mathrm{~kg}$, female $<18 \mathrm{~kg}$ & $85(47.5)$ & $45(37.5)$ & $40(67.8)$ & $<0.001$ \\
Usual gait speed $<1.0 \mathrm{~m} / \mathrm{s}$ & $47(26.3)$ & $14(11.7)$ & $33(55.9)$ & $<0.001$ \\
Sit to stand $\geq 12 \mathrm{~s}$ & $52(29.1)$ & $19(15.8)$ & $33(55.9)$ & $<0.001$ \\
SPPB $\leq 9$ & $41(22.9)$ & $11(9.2)$ & $30(50.9)$ & $<0.001$ \\
Low physical performance & $60(33.5)$ & $22(18.3)$ & $38(64.4)$ & $<0.001$ \\
SMI men $<7.0 \mathrm{~kg} / \mathrm{m}^{2}$, female $<5.7 \mathrm{~kg} / \mathrm{m}^{2}$ & $58(32.4)$ & $37(30.8)$ & $21(35.6)$ & 0.522 \\
\hline
\end{tabular}

Values are expressed as $n(\%)$ of patients. SMI, skeletal muscle mass; SPPB, short physical performance battery.

in the SARC-F < 4 group, as many as 28 (23.4\%) patients had been overlooked.

The sensitivity, specificity, positive predictive values, and negative predictive values for the AWGS2019 criteria are shown in Table 4. SARC-F had a low to moderate sensitivity (42.9\% for sarcopenia and $66.7 \%$ for severe sarcopenia), moderate specificity ( $70.8 \%$ for sarcopenia and $72.3 \%$ for severe sarcopenia), and relatively high negative predictive value $(76.7 \%$ for sarcopenia and $93.3 \%$ for severe sarcopenia). The AUCs of SARC-F for sarcopenia and severe sarcopenia were 0.57 (95\% confidence interval [CI]: $0.49-0.65)$ and 0.70 (95\% CI: 0.59-0.80), respectively.

\section{Discussion}

This cross-sectional study showed that 59 (33.0\%) patients on hemodialysis had an SARC-F score of $\geq 4$, and $49(27.4 \%)$ patients had sarcopenia according to the AWGS2019 criteria. Higher SARC-F scores were significantly associated with a higher likelihood of sarcopenia diagnosed using the AWGS2019 criteria and lower muscle strength and physical performance. We found that the SARC-F had moderate sensitivity and specificity with respect to the screening ability. In addition, in the SARC-F $<4$ group, 28 (23.4\%) patients with sarcopenia or severe sarcopenia diagnosed using the AWGS2019 criteria had been overlooked. Our results suggest that the SARC-F might be a useful tool to screen for low muscle strength and physical performance; however, it has limited screening ability for correctly identifying patients with sarcopenia among those undergoing hemodialysis. To the best of our knowledge, this is the first study to explore the validity of the SARC-F as a screening tool for sarcopenia, defined by the AWGS2019 criteria, in patients on hemodialysis.

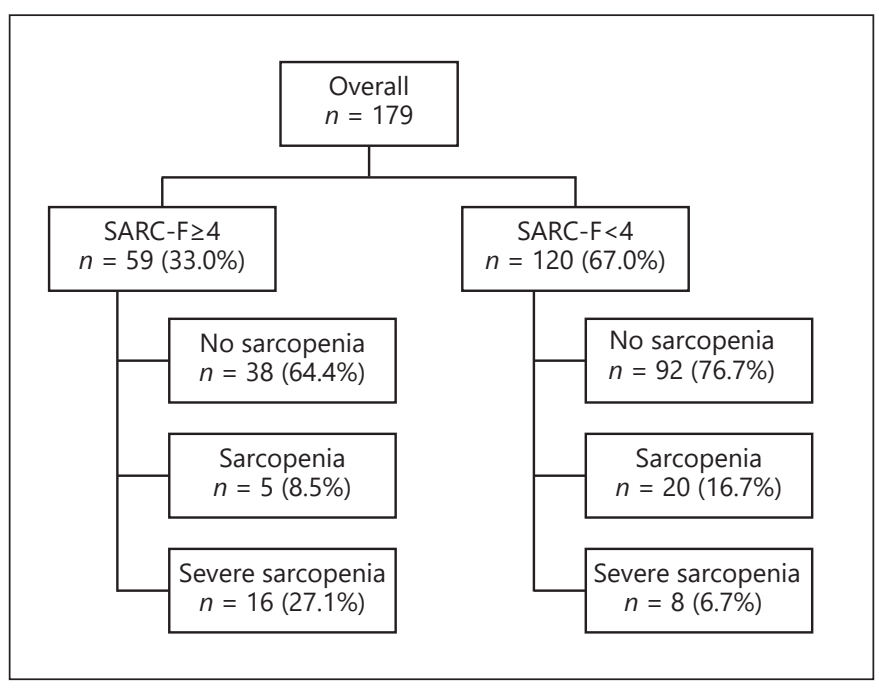

Fig. 1. The SARC-F and the AWGS2019 criteria diagram. SARC-F, Strength, Assistance in walking, Rise from a chair, Climb stairs, and Falls; AWGS2019, Asian Working Group for Sarcopenia.

In this study, the prevalence of sarcopenia or severe sarcopenia was $27.4 \%$, and the sensitivity and specificity of the SARC-F for sarcopenia were $42.9 \%$ and $70.8 \%$, respectively. Similar accuracy was reported in a previous study conducted among hospitalized patients, which showed that the prevalence of sarcopenia defined by the AWGS2019 criteria was 50.1-61.1\%, and the sensitivity and specificity were $49.1-62.3 \%$ and $71.4-81.2 \%$, respectively [30, 31]. In contrast, a study among communitydwelling older adults [32] reported that the prevalence of sarcopenia and severe sarcopenia was $22.8 \%$ and $6.8 \%$ and showed lower sensitivity (10.4-23.9\%) and higher specificity (92.8-93.6\%) values of the SARC-F than those obtained in our study. The probable reasons for the varying accuracy of the SARC-F across studies could be the 
Table 4. Indicators of performance of the SARC-F questionnaire with AWGS2019

\begin{tabular}{llllll}
\hline Definitions & Sensitivity, \% (95\% Cl) & Specificity, \% (95\% Cl) & PPV, \% (95\% Cl) & NPV, \% (95\% Cl) & AUC (95\% Cl) \\
\hline Sarcopenia & $42.9(28.8-57.8)$ & $70.8(62.2-78.4)$ & $35.6(23.6-49.1)$ & $76.7(68.1-83.9)$ & $0.57(0.49-0.65)$ \\
Severe sarcopenia & $66.7(44.7-84.4)$ & $72.3(64.5-79.1)$ & $27.1(16.4-40.3)$ & $93.3(87.3-97.1)$ & $0.70(0.59-0.80)$ \\
\hline
\end{tabular}

AUC, area under the curve; AWGS2019, Asian Working Group for Sarcopenia; Cl, confidence interval; NPV, negative predictive value; PPV, positive predictive value; SARC-F, Strength, Assistance in walking, Rise from a chair, Climb stairs, and Falls.

differences in the prevalence of sarcopenia in these populations. Generally, the sensitivity of a test is higher in a population with a higher prevalence of the target disease. In addition, the specificity tends to be lower in subjects with comorbidities (i.e., patients on hemodialysis and under hospital settings) due to a higher rate of false positives, depending on the severity of the comorbidities and disease-related pathology.

In the present study, the AUCs of SARC-F for the AWGS2019 were low, and the SARC-F $<4$ group included $28(23.4 \%)$ patients with sarcopenia or severe sarcopenia. The probable reason for this finding was that 24 patients in the SARC-F $<4$ group had cognitive decline (MMSE $<24)$. Previous studies have reported that patients with lower cognitive function are more likely to overestimate their mobility $[33,34]$. Therefore, cognitive decline might have led to the misclassification of these patients.

In the AWGS2019 guidelines, the SARC-F is suggested as an initial tool before completing a more thorough assessment for sarcopenia using more complicated physical performance tests and body composition assessments, which is the procedure for serial testing [35]. Given the present findings indicating that the SARC-F has a low to moderate sensitivity and specificity for the diagnosis of sarcopenia, it might be more practical to use it in combination with objective assessment measures (i.e., physical performance tests), rather than as a first-step screening tool, to ensure that sarcopenia is not overlooked in patients on hemodialysis.

This study has several limitations. First, the study targeted Japanese patients receiving hemodialysis at a single center. Furthermore, the BIA parameters may vary depending on lifestyle habits, such as diet; therefore, our results might have limited generalizability, and further validation studies across other Asian countries are necessary. Second, we assessed the muscle mass using BIA prior to a dialysis session, which could lead to an overestimation of the muscle mass in the setting of overhydration [36]; thus, the true prevalence of sarcopenia might have been underestimated. Future studies are necessary to val- idate sarcopenia defined by muscle mass measured after dialysis or by using different measuring equipment (i.e., dual-energy X-ray absorptiometry and magnetic resonance imaging). Third, in this study, gait speed was measured using the $10-\mathrm{m}$ walking test. The AWGS2019 recommends the measurement of the 6-m gait speed, and it cannot be ruled out that the results may differ from those measured using the 10 -m walk test. Fourth, cognitive impairment was assessed using the MMSE; however, the MMSE alone may not be sufficient for diagnosing cognitive impairment. Therefore, cognitive dysfunction may have been overestimated or underestimated. Finally, this study did not distinguish between patients with arteriovenous fistulas or catheters as vascular access for hemodialysis. The quality of dialysis and inflammatory status may have been affected, which in turn may have modified the degree of malnutrition and sarcopenia. Therefore, it cannot be ruled out that the investigation of the dialysis quality may have been insufficient.

\section{Conclusions}

We investigated the diagnostic ability of the SARC-F to diagnose sarcopenia and severe sarcopenia, as defined by the AWGS2019, among patients on hemodialysis. SARC-F $\geq 4$ had low to moderate sensitivity and specificity for sarcopenia. In clinical practice, careful interpretation might be necessary to diagnose sarcopenia using only the SARC-F in patients on dialysis. Further, as the working groups for sarcopenia suggest, a comprehensive screening with objective assessments, in addition to the SARC-F, might be necessary.

\section{Acknowledgments}

The authors thank all patients who participated in this study and the HD treatment staff at the Sagami Circulatory Organ Clinic for their contributions to this study. 


\section{Statement of Ethics}

This study was performed according to the ethical principles of the Declaration of Helsinki and was approved by the research ethics committee. The patients provided written informed consent prior to study participation.

\section{Conflict of Interest Statement}

The authors declare no conflicts of interest.

\section{Funding Sources}

This study was supported by the Japan Society for the Promotion of Science (JSPS) KAKENHI, Grant No. JP19K11372, received by Dr. Matsunaga.

\section{Author Contributions}

K.I. and S.Y. contributed to research idea and study design; K.I., S.Y., Y.S., M.H., and Y.S. contributed to data acquisition; K.I., S.Y., Y.S., R.M., M.H., Y.S., and A.M. contributed to data analysis/interpretation; K.I. and S.Y. contributed to statistical analysis; Y.S., R.M., A.Y., and A.M. contributed to supervision or mentorship. Each author contributed important intellectual content during manuscript drafting or revision, accepts personal accountability for the author's own contributions, and agrees to ensure that questions pertaining to the accuracy or integrity of any portion of the work are appropriately investigated and resolved.

\section{Data Availability Statement}

The data that support the findings of this study are not publicly available due to their containing information that could compromise the privacy of research participants.

\section{References}

1 Chen LK, Liu LK, Woo J, Assantachai P, Auyeung TW, Bahyah KS, et al. Sarcopenia in Asia: consensus report of the Asian Working Group for Sarcopenia. J Am Med Dir Assoc. 2014 Feb;15(2):95-101.

2 Chen LK, Woo J, Assantachai P, Auyeung TW, Chou MY, Iijima K, et al. Asian Working Group for Sarcopenia: 2019 consensus update on sarcopenia diagnosis and treatment. J Am Med Dir Assoc. 2020 Mar;21(3):300-7.e2.

3 Kim JC, Kalantar-Zadeh K, Kopple JD. Frailty and protein-energy wasting in elderly patients with end stage kidney disease. J Am Soc Nephrol. 2013 Feb;24(3):337-51.

4 Woo J, Leung J, Morley JE. Defining sarcopenia in terms of incident adverse outcomes. J Am Med Dir Assoc. 2015 Mar;16(3):247-52.

5 Chang KV, Hsu TH, Wu WT, Huang KC, Han DS. Association between sarcopenia and cognitive impairment: a systematic review and meta-analysis. J Am Med Dir Assoc. 2016 Dec 1;17(12):1164.e7-15.

6 Chang KV, Hsu TH, Wu WT, Huang KC, Han DS. Is sarcopenia associated with depression? A systematic review and meta-analysis of observational studies. Age Ageing. 2017 Sep 1;46(5):738-46.

7 Szlejf C, Suemoto CK, Brunoni AR, Viana MC, Moreno AB, Matos SMA, et al. Depression is associated with sarcopenia due to low muscle strength: results from the ELSA-Brasil Study. J Am Med Dir Assoc. 2019 Dec;20(12): 1641-6.

8 Yeung SSY, Reijnierse EM, Pham VK, Trappenburg MC, Lim WK, Meskers CGM, et al. Sarcopenia and its association with falls and fractures in older adults: a systematic review and meta-analysis. J Cachexia Sarcopenia Muscle. 2019 Jun;10(3):485-500.

9 Kittiskulnam P, Carrero JJ, Chertow GM, Kaysen GA, Delgado C, Johansen KL. Sarco- penia among patients receiving hemodialysis: weighing the evidence. J Cachexia Sarcopenia Muscle. 2017 Feb;8(1):57-68.

10 Isoyama $\mathrm{N}$, Qureshi AR, Avesani CM, Lindholm $\mathrm{B}$, Bàràny $\mathrm{P}$, Heimbürger $\mathrm{O}$, et al. Comparative associations of muscle mass and muscle strength with mortality in dialysis patients. Clin J Am Soc Nephrol. 2014 Oct 7; 9(10):1720-8.

11 Kim JK, Choi SR, Choi MJ, Kim SG, Lee YK, Noh JW, et al. Prevalence of and factors associated with sarcopenia in elderly patients with end-stage renal disease. Clin Nutr. 2014 Feb;33(1):64-8.

12 Lamarca F, Carrero JJ, Rodrigues JC, Bigogno FG, Fetter RL, Avesani CM. Prevalence of sarcopenia in elderly maintenance hemodialysis patients: the impact of different diagnostic criteria. J Nutr Health Aging. 2014 Jul;18(7): $710-7$.

13 Mori K, Nishide K, Okuno S, Shoji T, Emoto $\mathrm{M}$, Tsuda A, et al. Impact of diabetes on sarcopenia and mortality in patients undergoing hemodialysis. BMC Nephrol. 2019 Mar 28; 20(1): 105 .

14 Kittiskulnam P, Chertow GM, Carrero JJ, Delgado C, Kaysen GA, Johansen KL. Sarcopenia and its individual criteria are associated, in part, with mortality among patients on hemodialysis. Kidney Int. 2017 Jul;92(1):23847.

15 Alston H, Burns A, Davenport A. Loss of appendicular muscle mass in haemodialysis patients is associated with increased self-reported depression, anxiety and lower general health scores. Nephrology. 2018 Jun;23(6): 546-51.

16 Delgado C, Johansen KL. Deficient counseling on physical activity among nephrologists. Nephron Clin Pract. 2010;116(4): c330-6.
17 Wang XX, Lin ZH, Wang Y, Xu MC, Kang $\mathrm{ZM}$, Zeng W, et al. Motivators for and barriers to exercise rehabilitation in hemodialysis centers: a multicenter cross-sectional survey. Am J Phys Med Rehabil. 2020 May;99(5):424-9.

18 Cruz-Jentoft AJ, Bahat G, Bauer J, Boirie Y, Bruyère $O$, Cederholm $T$, et al. Sarcopenia: revised European consensus on definition and diagnosis. Age Ageing. 2019 Jan 1;48(1):1631.

19 Malmstrom TK, Morley JE. SARC-F: a simple questionnaire to rapidly diagnose sarcopenia. J Am Med Dir Assoc. 2013 Aug; 14(8):531-2.

20 Morley JE, Cao L. Rapid screening for sarcopenia. J Cachexia Sarcopenia Muscle. 2015 Dec;6(4):312-4.

21 Malmstrom TK, Miller DK, Simonsick EM, Ferrucci L, Morley JE. SARC-F: a symptom score to predict persons with sarcopenia at risk for poor functional outcomes. J Cachexia Sarcopenia Muscle. 2016 Mar;7(1):28-36.

22 Tanaka S, Kamiya K, Hamazaki N, Matsuzawa R, Nozaki K, Ichinosawa Y, et al. SARC-F questionnaire identifies physical limitations and predicts post discharge outcomes in elderly patients with cardiovascular disease. JCSM Clinical Reports. 2018;3(1):1-11.

23 Yamamoto S, Matsuzawa R, Harada M, Watanabe T, Shimoda T, Suzuki Y, et al. SARC-F questionnaire: rapid and easy tool for identifying physical limitations in hemodialysis patients. JCSM Clin Rep. 2019;4(1):1-12.

24 Liu J, Huang Z, Gilbertson DT, Foley RN, Collins AJ. An improved comorbidity index for outcome analyses among dialysis patients. Kidney Int. 2010 Jan;77(2):141-51.

25 Yamada K, Furuya R, Takita T, Maruyama Y, Yamaguchi Y, Ohkawa S, et al. Simplified nutritional screening tools for patients on maintenance hemodialysis. Am J Clin Nutr. 2008 Jan;87(1):106-13. 
26 Lopes AA, Albert JM, Young EW, Satayathum S, Pisoni RL, Andreucci VE, et al. Screening for depression in hemodialysis patients: associations with diagnosis, treatment, and outcomes in the DOPPS. Kidney Int. 2004 Nov;66(5):2047-53.

27 Tsoi KK, Chan JY, Hirai HW, Wong SY, Kwok TC. Cognitive tests to detect dementia: a systematic review and meta-analysis. JAMA Intern Med. 2015 Sep;175(9):1450-8.

28 Guralnik JM, Simonsick EM, Ferrucci L, Glynn RJ, Berkman LF, Blazer DG, et al. A short physical performance battery assessing lower extremity function: association with self-reported disability and prediction of mortality and nursing home admission. J Gerontol. 1994 Mar;49(2):M85-94.

29 Woo J, Leung J, Morley JE. Validating the SARC-F: a suitable community screening tool for sarcopenia? J Am Med Dir Assoc. 2014 Sep;15(9):630-4.
30 Nguyen TN, Nguyen AT, Khuong LQ, Nguyen TX, Nguyen HTT, Nguyen TTH, et al. Reliability and validity of SARC-F questionnaire to assess sarcopenia among vietnamese geriatric patients. Clin Interv Aging. 2020;15: 879-86.

31 Ishida Y, Maeda K, Nonogaki T, Shimizu A, Yamanaka Y, Matsuyama R, et al. SARC-F as a screening tool for sarcopenia and possible sarcopenia proposed by AWGS 2019 in hospitalized older adults. J Nutr Health Aging. 2020 Aug 11;24:1053-60.

32 Kim M, Won CW. Sarcopenia in Korean community-dwelling adults aged 70 years and older: application of screening and diagnostic tools from the Asian Working Group for Sarcopenia 2019 update. J Am Med Dir Assoc. 2020 Jun;21(6):752-8.
33 Fischer BL, Gleason CE, Gangnon RE, Janczewski J, Shea T, Mahoney JE. Declining cognition and falls: role of risky performance of everyday mobility activities. Phys Ther. 2014 Mar;94(3):355-62.

34 Roedersheimer KM, Pereira GF, Jones CW, Braz VA, Mangipudi SA, Platts-Mills TF. Selfreported versus performance-based assessments of a simple mobility task among older adults in the emergency department. Ann Emerg Med. 2016 Feb;67(2):151-6.

35 Dent E, Morley JE, Cruz-Jentoft AJ, Arai H, Kritchevsky SB, Guralnik J, et al. International clinical practice guidelines for sarcopenia (ICFSR): screening, diagnosis and management. J Nutr Health Aging. 2018;22(10): 1148-61.

36 Janssen I, Heymsfield SB, Baumgartner RN, Ross R. Estimation of skeletal muscle mass by bioelectrical impedance analysis. J Appl Physiol. 2000 Aug;89(2):465-71. 